\title{
The Quantum Redshift Effect of Photon
}

\author{
Zhenglong Xu \\ Wuzhen High School, Tongxiang, China \\ Email: zhl-xu@126.com
}

How to cite this paper: Xu, Z.L. (2021) The Quantum Redshift Effect of Photon. Journal of Modern Physics, 12, 2003-2030. https://doi.org/10.4236/jmp.2021.1214115

Received: November 24, 2021

Accepted: December 28, 2021

Published: December 31, 2021

Copyright (c) 2021 by author(s) and Scientific Research Publishing Inc. This work is licensed under the Creative Commons Attribution International License (CC BY 4.0).

http://creativecommons.org/licenses/by/4.0/

\begin{abstract}
By applying the relevant contents of time-dependent perturbation theory in present quantum mechanics, this paper operates the Hamiltonian operator on the existing steady-state wave function of the hydrogen atoms, creates the formula of the average energy loss per collision of a single photon with a neutral hydrogen atom. Then, by operating the Hamiltonian operator on the wave function of a helium atom, the expression of photon energy loss is extended to each bound electron in the neutral atoms of the general element. Applying theoretical derivation, it concludes that the photon's energy, momentum, and frequency decrease with distance in an exponential attenuation law, or the wavelength increases with distance in an exponential growth law during the propagation in intergalactic space, the photons collide with neutral atoms in the medium. Compared with Hubble redshift law, we discover that these laws conform to the so-called Hubble redshift or cosmological redshift in form and quantity. Therefore, this paper reveals a new physical mechanism-the quantum redshift effect of the photon in astronomy, astrophysics, and cosmology, thus challenging Hubble's law and the Big Bang theory.
\end{abstract}

\section{Keywords}

Hamiltonian Operator, Perturbation Theory, Hubble Redshift, Cosmological Redshift, Quantum Redshift Effect, Hubble Constant

\section{Introduction}

The Big Bang theory considered the "Redshift" be evidence that the universe is expanding. Whether the redshift is evidence has always been a matter of great debate. The reason for the controversy is that after that explanation, few people have done in-depth research on the physical mechanism of the origin of the redshift, and physicists do not seem to be interested in this question, but rather in dark matter and dark energy. How photons travel through intergalactic space with a redshift is a mystery. Since discovered quasars in the 1950s, the redshift is 
not entirely consistent with Hubble's law and the so-called cosmological redshift, leading physicists to revisit the origin of redshifts. There have been many theories in history, such as tired light theory, photon aging theory, intrinsic redshift theory, physical constant change theory, new tired light theory. But no one can fully reveal its physical mechanism to convince the Big Bang theorists. So, what way can solve this mystery?

\section{The Collision of a Single Photon with a Free Electron}

This section attempts to explore the physical mechanism of the origin of redshift employing classical electrodynamics. There are different opinions in astrophysics about whether the intergalactic space medium is neutral gas or plasma gas. Assuming the intergalactic medium is thin plasma rather than a continuum. The electrodynamic treatment of electromagnetic wave propagation in continuous media is not adopted. This paper uses the term "photon" rather than "light"; and utilizes the ideas of the size and shape of a single photon [1]. Suppose a photon collides with an electron in a plasma, how much energy lost the photon does, and what direction the photon travels.

\subsection{Relationship between Compton Scattering and Thomson Scattering}

Suppose that photons travel from distant galaxies to Earth through intergalactic space, where the medium is a thin plasma that is consisted of electrons and protons. Photons can only collide with a free electron or a free ion, but it is almost impossible to with both simultaneously. Since there is no mutual constraint between electrons and nuclei, the way to deal with photons colliding with charged particles is classical electrodynamics rather than quantum mechanics.

In the energy calculation, the energy lost by the collision of photons with free electrons is much larger than that lost by the collision of free protons. Therefore, only that between the photon and the free-electron is considered, not between photon and proton.

When a single photon collides with a free electron, one of the effects may occur. The first effect is the Compton effect and the second effect is the Thomson effect.

The probability of a Compton effect occurring in a collision between a single photon and a free electron is [1]

$$
p_{C}=\frac{\pi}{2} \frac{\lambda_{C}}{\lambda}
$$

And the probability of the Thomson effect is

$$
p_{T}=\frac{2 \lambda-\pi \lambda_{C}}{2 \lambda}
$$

Here, $\lambda_{C}$ is the Compton wavelength of an electron, and $\lambda$ is the wavelength of a photon. 
Apparently, for photons in the optical band, the wavelength is longer than the Compton wavelength, i.e., $\lambda \gg \lambda_{C}$. Therefore, the probability of the Compton effect is very low, but the energy lost in each collision is relatively high. When photons in the optical band collide with free electrons, the probability of the Thomson effect is much higher than that of Compton scattering, the energy lost by photons in each collision is relatively low.

The paper "the size and shape of a single photon" deeply discussed the Compton effect, which found that the condition for the Compton effect is: First, the photon wavelength is small enough to meet $\lambda \leq \pi \lambda_{C} / 2$; Second, although it does not meet $\lambda \leq \pi \lambda_{C} / 2$, the electron is collided by the edge of the photon. Under such conditions, the interaction time cannot reach the photon vibration period that the electron ejects by the photon electric field. In Compton scattering, both the electron and the photon change direction. Therefore, the photon no longer travels in a straight line.

In Compton scattering, if the interaction time between electron and photon reaches one period, $T$, there is no Compton effect. In this case, the Thomson effect will occur.

\subsection{Thomson Effect of Single Photon-Single Electron}

This section focuses on the Thomson effect when a single photon collides with a free electron.

Suppose the photon propagates along the $\mathrm{Z}$-axis at the velocity $c$, the electric field vibrates along the $\mathrm{X}$-axis, and the mass of the electron is $m_{e}$. The charge of the electron is $-e$. The electric field strength of photon is

$$
E=E_{0} \sin \omega\left(t-\frac{z}{c}\right)
$$

where $E_{0}$ is the amplitude of photon electric field strength, $\omega$ is the angular frequency of photon electric field vibration, and $t$ is time.

Assuming that the electron initially rests at the origin of the coordinate OXYZ, the initial position is $x_{0}=0$, the initial speed is $\dot{x}_{0}=0$, omitting $z / c=0$ in the brackets of the sinusoidal function, the electric field force of the electron acted by the photon is

$$
F_{e}=-e E_{0} \sin \omega t
$$

When an electron vibrates in the electric field of a photon, electromagnetic radiation will occur. In turn, the radiation acts on the electron, causing the electron to subject a damping force proportional to the moving velocity $\dot{x}$

$$
f=-\gamma_{0} m_{e} \dot{x}
$$

where $\gamma_{0}$ is the damping coefficient of the electron

$$
\gamma_{0}=\frac{e^{2} \omega^{2}}{6 \pi \varepsilon_{0} m_{e} c^{3}}
$$

where $\varepsilon_{0}$ is the vacuum dielectric constant. 
The resultant force on the electron is

$$
F=m_{e} \ddot{x}=-e E_{0} \sin \omega t-\gamma_{0} m_{e} \dot{x}
$$

Thus, can list the following equation

$$
\left\{\begin{array}{l}
\ddot{x}+\gamma_{0} \dot{x}=-\frac{e E_{0}}{m_{e}} \sin \omega t \\
x_{0}=0 ; \dot{x}_{0}=0
\end{array}\right.
$$

By solving this equation, the velocity of the electron is

$$
\dot{x}=\frac{e E_{0}}{m_{e}\left(\omega^{2}+\gamma_{0}^{2}\right)}\left[\left(\gamma_{0} \sin \omega t+\omega \cos \omega t\right)-\omega \mathrm{e}^{-\gamma_{0} t}\right]
$$

The displacement of the electron is

$$
x=-\frac{e E_{0}}{m_{e} \omega \gamma_{0}\left(\omega^{2}+\gamma_{0}^{2}\right)}\left[\left(\omega^{2}-\gamma_{0}^{2}\right)-\left(\omega^{2} \mathrm{e}^{-\gamma_{0} t}-\gamma_{0}^{2} \cos \omega t+\omega \gamma_{0} \sin \omega t\right)\right]
$$

The work done by photon to the electron is

$$
\begin{aligned}
W & =\int_{0}^{X} F_{e} \mathrm{~d} x=\int_{0}^{T} F \dot{x} \mathrm{~d} t \\
& =\frac{e^{2} E_{0}^{2}}{m_{e}\left(\omega^{2}+\gamma_{0}^{2}\right)} \int_{0}^{T} \sin \omega t\left[\left(\gamma_{0} \sin \omega t+\omega \cos \omega t\right)-\omega \mathrm{e}^{-\gamma_{0} t}\right] \mathrm{d} t \\
& =\frac{e^{2} E_{0}^{2}}{m_{e}\left(\omega^{2}+\gamma_{0}^{2}\right)}\left[\frac{1}{2} \gamma_{0} T+\frac{\omega^{2}}{\omega^{2}+\gamma_{0}^{2}}\left(1-\mathrm{e}^{-\gamma_{0} T}\right)\right]
\end{aligned}
$$

where $T$ is the period of photon vibration [1].

Under the condition of $\gamma_{0} \ll \omega$ and $\gamma_{0} \ll \omega_{0}$, take the approximate $\mathrm{e}^{-\gamma_{0} T} \approx 1-\gamma_{0} T$, the above formula becomes

$$
\begin{aligned}
W & =\frac{e^{2} E_{0}^{2}}{m_{e}\left(\omega^{2}+\gamma_{0}^{2}\right)}\left[\frac{1}{2} \gamma_{0} T+\frac{\omega^{2} \gamma_{0} T}{\omega^{2}+\gamma_{0}^{2}}\right] \\
& =\frac{3 e^{2} E_{0}^{2}}{2 m_{e} \omega^{2}} \gamma_{0} T
\end{aligned}
$$

Use Equation (6), and the above equation becomes

$$
\begin{aligned}
W & =2 \pi \alpha \frac{(\hbar \omega)^{3}}{\left(m_{e} c^{2}\right)^{2}} \\
& =3 \frac{\sigma_{T}}{\sigma_{P}} \cdot \hbar \omega
\end{aligned}
$$

Here utilizing the following relationship:

The relationship between the electric field strength of a single photon and the photon frequency [1]

$$
e E_{0}=\frac{\hbar \omega^{2}}{c}
$$


The fine structure constant

$$
\alpha=\frac{e^{2}}{4 \pi \varepsilon_{0} \hbar c}
$$

The classical electron radius formula

$$
r_{e}=\frac{e^{2}}{4 \pi \varepsilon_{0} m_{e} c^{2}}
$$

The Thomson scattering cross-sectional area of classical electron

$$
\sigma_{T}=\frac{8 \pi}{3} r_{e}^{2}
$$

The cross-sectional area of linearly polarized photon [1]

$$
\sigma_{P}=\frac{\alpha}{\pi^{2}} \lambda^{2}
$$

In the above formulas, $\hbar$ is the reduced Planck constant.

Equation $(11-\mathrm{g})$ shows that the work done by a photon to an electron is equal to the ratio of three times the cross-sectional area of the electron to the crosssectional area of the photon multiplied by photon energy. Every time a photon and a free electron occur Thomson scattering, the energy lost by the photon is $W=\left(3 \sigma_{T} / \sigma_{p}\right) \hbar \omega$. It means that the energy lost by the photon is that intercepted by the electron.

In macroscopic electrodynamics, express the formula of Thomson scattering of a beam of light by a single electron as the power of electron scattering as the following:

$$
P_{T}=\sigma_{T} I_{0}
$$

where $I_{0}$ is the intensity of the incident beam of light. In classical electrodynamics, the light intensity $I_{0}$ is independent of photon frequency.

The difference between Equations (11) and (17) represents the difference between a single photon and a beam of light.

Equation (9) shows that when the photon interacts with the electron for a period $T$, the electron gets the recoil velocity or the recoil momentum in the X-direction; in turn, the photon momentum also changes in the X-direction, which means that the photon no longer propagates in a straight line.

Summary:

1) Because Compton scattering and Thomson scattering will change the direction of photon motion, once Compton scattering or Thomson scattering occurs on the path of propagation to the Earth, it cannot be received by observers on the Earth. Therefore, Compton scattering and Thomson scattering cannot be the cause of extragalactic galaxy redshift.

2) The occurrence of the Compton effect and Thomson effect depends on plasma, so it can infer that the neutral gas dominates the intergalactic medium rather than plasma. 


\section{Collision of a Single Photon with a Hydrogen Atom}

\subsection{Size Comparison between Photon and Atom}

Photons of different wavelengths have different sizes. For studying the energy loss in the collision between a single photon and an atom, it is necessary to understand several typical photon size parameters. All photons mentioned in this paper are linearly polarized photons.

Here, the electric field size of a photon refers to the length of the photon along the electric field direction, and the formula is [1]

$$
L_{E}=\frac{2}{\pi} \lambda
$$

The photon cross-sectional area formula is as Equation (16).

Photons in Table 1 refer to:

1) The microwave corresponding to the maximum energy distribution of the cosmic microwave background;

2) Infrared corresponding to the maximum energy distribution of human thermal radiation;

3) The most sensitive light of the human eye;

4) Ultraviolet light with the shortest wavelength;

5) An X-ray used in the Compton experiment [2];

6) $\gamma$-rays produced by electron pair annihilation;

7) $\gamma$-rays produced by proton pair annihilation;

As well known that the order of magnitude of the diameter of an atom is $10^{-10}$ $-10^{-9} \mathrm{~m}$. The table indicates that the electric field size of ultraviolet light with the shortest wavelength is greater than the atomic diameter. The electric field size of photons with a longer wavelength than ultraviolet light with the shortest wavelength is larger than the atom diameter.

According to the comparison between the size of the photon electric field and the atom diameter, photons are divided into two categories. Photons whose electric field size is larger than the atom diameter are called soft photons, and photons whose electric field size is smaller than the atom diameter are called hard photons.

Table 1. The wavelength, electric field size, and cross-sectional area of some photons.

\begin{tabular}{llll}
\hline Photon & Wavelength/m & Electric field size $/ \mathrm{m}$ & Cross-sectional area $/ \mathrm{m}^{2}$ \\
\hline 1 & $7.4 \times 10^{-2}$ & $4.7 \times 10^{-2}$ & $4.0 \times 10^{-8}$ \\
2 & $1.0 \times 10^{-5}$ & $6.4 \times 10^{-4}$ & $7.4 \times 10^{-14}$ \\
3 & $5.3 \times 10^{-7}$ & $3.4 \times 10^{-7}$ & $2.1 \times 10^{-16}$ \\
4 & $1.0 \times 10^{-8}$ & $6.4 \times 10^{-9}$ & $7.4 \times 10^{-20}$ \\
5 & $7.1 \times 10^{-11}$ & $4.5 \times 10^{-11}$ & $3.7 \times 10^{-24}$ \\
6 & $2.4 \times 10^{-12}$ & $1.5 \times 10^{-12}$ & $4.2 \times 10^{-27}$ \\
7 & $1.3 \times 10^{-15}$ & $8.3 \times 10^{-14}$ & $1.2 \times 10^{-33}$ \\
\hline
\end{tabular}




\subsection{Soft Photons and Hard Photons Collide with Atoms to Produce Different Effects}

Soft and hard photons have different physical effects when they collide with atoms.

Because the electric field size of a soft photon is greater than that of an atom, when a photon collides with the atom, the whole will be immersed in the photon electromagnetic field, resulting in the atomic nucleus and electrons in the atom being affected by the photon electromagnetic field simultaneously. As shown in Figure 1 is the collision model of soft photon-atom.

Because the electric field size of the photon is shorter than that of the atom when a hard photon collides with it, the photon can't be to collide with the whole atom but enter its interior. The photon can collide only with one of the nucleus or electrons in the atom, and it is impossible to collide with the electron and nucleus simultaneously. As shown in Figure 2 is the collision model of hard photon-atom.

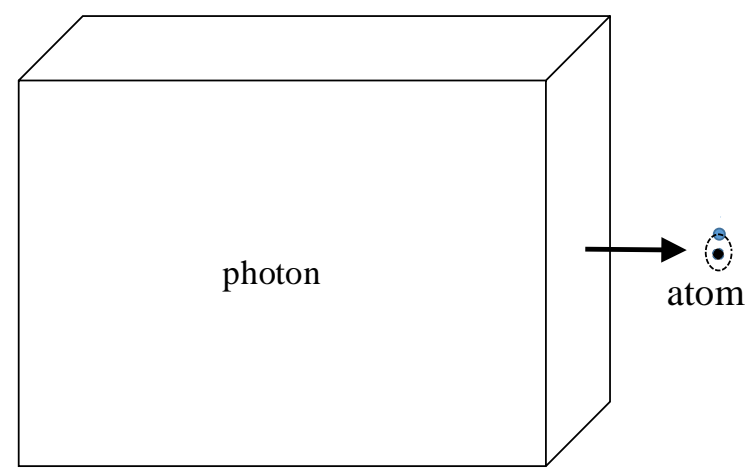

Figure 1. A soft photon collides with an atom.

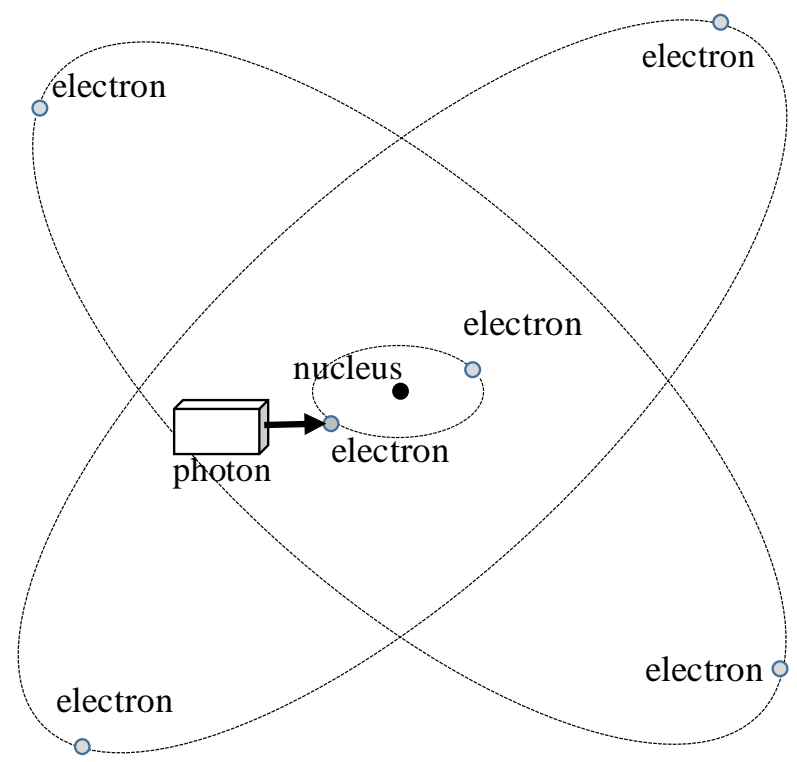

Figure 2. A hard photon enters into an atom and strikes one of electrons. 
In this way, when a photon enters the atom, one of the following two situations may occur:

The first is Compton scattering, in which the propagation direction of photons will change.

The second is that if the cross-sectional area of the hard photon is small enough, the photon may penetrate atoms unimpeded without colliding with any kinds of particles.

Summary:

When a single photon collides with an atom, it is necessary to distinguish between soft photons and hard photons. Only soft photons can interact with the nucleus and extranuclear electrons simultaneously.

\section{The Energy Loss in a Collision between the Photon and Hydrogen Atom}

The focus of the following study is the collision of a soft photon with a hydrogen atom.

Why study the collision of a soft photon with a hydrogen atom? It is for two reasons:

First, only the soft photon can collide with the nuclei and the electron simultaneously because of its large size.

Second, in quantum mechanics textbooks, the only atom studied is hydrogen, and the treatment of helium and the higher elements is approximate.

\subsection{Correction of Hamiltonian}

Classical electrodynamics can deal with the collision between photons and free electrons. Quantum mechanics can deal with that between a photon with bound electrons.

When there is no external electromagnetic field, extranuclear electron of a hydrogen atom are only affected by the electric field of the nucleus, and the Hamiltonian can express as

$$
\mathcal{H}_{0}=\frac{\boldsymbol{P}_{0}^{2}}{2 m_{e}}+U(r)
$$

where $\boldsymbol{P}_{0}$ is the momentum of the extranuclear electron and $U(r)$ is the electric potential energy of the electron in the electric field of the atomic nucleus, which can express as

$$
U(r)=-\frac{1}{4 \pi \varepsilon_{0}} \frac{e^{2}}{r}
$$

When the photon electromagnetic field suddenly acts on the hydrogen atom, the nucleus and the extranuclear electron are affected by the photon electromagnetic field simultaneously, which will change their kinetic energy and potential energy. Since the mass of the hydrogen nucleus is 1836 times that of the electron, the kinetic energy it gets is negligible, so only the electron is consi- 
dered.

Suppose the functions of electric field strength and magnetic induction strength of electromagnetic wave of a photon be

$$
\left\{\begin{array}{l}
\boldsymbol{E}=E_{0} \sin \omega\left(t-\frac{z}{c}\right) \boldsymbol{e}_{x} \\
\boldsymbol{B}=B_{0} \sin \omega\left(t-\frac{z}{c}\right) \boldsymbol{e}_{y}
\end{array}\right.
$$

whose vector potential $\boldsymbol{A}$ conforms to

$$
\left\{\begin{array}{l}
\boldsymbol{E}=-\frac{\partial \boldsymbol{A}}{\partial t} \\
\boldsymbol{B}=\nabla \times \boldsymbol{A}
\end{array}\right.
$$

Regardless of the integral constant, integrating can obtain

$$
\begin{aligned}
\boldsymbol{A} & =-\int \boldsymbol{E} \mathrm{d} t=-E_{0} \boldsymbol{e}_{x} \int \sin \omega\left(t-\frac{z}{c}\right) \mathrm{d} t \\
& =\frac{E_{0}}{\omega} \cos \omega\left(t-\frac{z}{c}\right) \boldsymbol{e}_{x} \\
& =\frac{E_{0}}{2 \omega}\left[\mathrm{e}^{i \omega\left(t-\frac{z}{c}\right)}+\mathrm{e}^{-i \omega\left(t-\frac{z}{c}\right)}\right] \boldsymbol{e}_{x}
\end{aligned}
$$

The sudden appearance of the electromagnetic field of the photon causes two changes of extranuclear electrons:

The first is the change of electron momentum caused by the vector potential of the photon electromagnetic field

$$
\Delta \boldsymbol{P}^{\prime}=-e \boldsymbol{A}
$$

Acted by the photon electromagnetic field, the momentum of the electron becomes

$$
\boldsymbol{P}=\boldsymbol{P}_{0}+\Delta \boldsymbol{P}^{\prime}=\boldsymbol{P}_{0}-e \boldsymbol{A}
$$

The second is that the scalar potential of the photon electric field causes the change of the electric potential energy of the electron:

$$
V(r)=-e \boldsymbol{E} \cdot \boldsymbol{r}=-e E_{0} x \sin \omega\left(t-\frac{z}{c}\right)
$$

Thus, when the photon electromagnetic field acts on the hydrogen atom, the Hamiltonian is

$$
\mathcal{H}=\frac{1}{2 m_{e}}\left(\boldsymbol{P}_{0}-e \boldsymbol{A}\right)^{2}+U(r)+V(r)
$$

In addition, the orbital magnetic moment and spin magnetic moment of the electron will also cause the change of potential energy in the photon magnetic field. This potential-energy term can incorporate into the term $V(r)$. The calculation result can show the splitting of atomic energy-level, that is, the Zeeman effect. Quantum mechanics textbooks have a detailed discussion [3] (1), which omits 
here.

Discussing the correction term $V(r)$ in detail in quantum mechanics textbooks clarifies the physical mechanism by which photons are absorbed by atoms as a whole or atoms are excited by photons to emit photons. The change in momentum $\Delta \boldsymbol{P}^{\prime}=-e \boldsymbol{A}$, which is overlooked usually in textbooks, hides a profound secret.

\subsection{Basic Theory of Hamiltonian Operator Operation}

According to the general textbook of quantum mechanics [4], the Hamiltonian operator corresponding to the Hamiltonian of Equation (27) is

$$
\begin{aligned}
\hat{\mathcal{H}} & =\frac{1}{2 m_{e}}\left(\hat{\boldsymbol{P}}_{0}-e \boldsymbol{A}\right)^{2}+\hat{U}(r)+\hat{V}(r) \\
& =\frac{1}{2 m_{e}} \hat{\boldsymbol{P}}_{0}^{2}+\hat{U}(r)+\hat{V}(r)-\frac{e}{2 m_{e}}\left(\hat{\boldsymbol{P}}_{0} \cdot \boldsymbol{A}-\boldsymbol{A} \cdot \hat{\boldsymbol{P}}_{0}\right)+\frac{e^{2}}{2 m_{e}} \boldsymbol{A}^{2}
\end{aligned}
$$

where the momentum operator is

$$
\hat{\boldsymbol{P}}_{0}=-i \hbar \nabla
$$

Generally speaking, $\hat{\boldsymbol{P}}_{0}$ does not commute with $\boldsymbol{A}$, so there is a relationship

$$
\hat{\boldsymbol{P}}_{0} \cdot \boldsymbol{A}-\boldsymbol{A} \cdot \hat{\boldsymbol{P}}_{0}=-i \hbar \nabla \cdot \boldsymbol{A}
$$

However, for photons, since the electromagnetic wave is a transverse wave, applying Equation (23-b) can obtain $\nabla \cdot \boldsymbol{A}=0$, then $\hat{\boldsymbol{P}}_{0}$ commutes with $\boldsymbol{A}$, so Equation (30) becomes $\hat{\boldsymbol{P}}_{0} \cdot \boldsymbol{A}-\boldsymbol{A} \cdot \hat{\boldsymbol{P}}_{0}=0$ that the Hamiltonian operator (28) can express as

$$
\hat{\mathcal{H}}=\frac{1}{2 m_{e}} \hat{\boldsymbol{P}}_{0}^{2}+U(r)+V(r)-\frac{e}{m_{e}} \boldsymbol{A} \cdot \hat{\boldsymbol{P}}_{0}+\frac{e^{2}}{2 m_{e}} \boldsymbol{A}^{2}
$$

Group this representation into four parts:

The first part is

$$
\hat{\mathcal{H}}_{0}=\frac{1}{2 m_{e}} \hat{\boldsymbol{P}}_{0}^{2}+\hat{U}(r)
$$

It is the Hamiltonian operator of the Hydrogen atom when it is not affected by the photon electromagnetic field, and it is the Eigen-operator of the hydrogen atomic system.

The second part is

$$
\hat{\mathcal{H}}_{1}^{\prime}=\hat{V}(r)=-e E_{0} \hat{x} \sin \omega\left(t-\frac{Z}{c}\right)
$$

It is the potential energy of an atom as an electric dipole, and this term takes as a perturbation term. This paper refers to herein as the first correction term.

The third part is

$$
\hat{\mathcal{H}}_{2}^{\prime}=-\frac{e}{m_{e}} \boldsymbol{A} \cdot \hat{\boldsymbol{P}}_{0}
$$


This term is proportional to the dot product of the momentum vector of the extranuclear electron caused by the action of the photon electromagnetic field and the Eigen-momentum vector, which is called the second correction term in this paper.

Since the photon propagates along the $\mathrm{Z}$-axis, the photon electric field vibrates along the $\mathrm{X}$-axis, as described in Equation (21-1); The vector potential of the photon vibrates along the $\mathrm{X}$-axis, as described in Equation (23), so that

$$
\boldsymbol{A} \cdot \hat{\boldsymbol{P}}_{0}=A \hat{P}_{x}=A\left(-i \hbar \frac{\partial}{\partial x}\right)
$$

For a photon whose wavelength far exceeds the atomic radius, the time difference $\omega z / c$ can ignore, so that

$$
\boldsymbol{A}=\frac{E_{0}}{2 \omega}\left(\mathrm{e}^{\mathrm{i} \omega t}+\mathrm{e}^{-i \omega t}\right) \boldsymbol{e}_{x}
$$

Combining (35) and (36) obtain

$$
\boldsymbol{A} \cdot \hat{\boldsymbol{P}}_{0}=\frac{E_{0}}{2 \omega}\left(\mathrm{e}^{i \omega t}+\mathrm{e}^{-i \omega t}\right)\left(-i \hbar \frac{\partial}{\partial x}\right)
$$

Substituting (37) into (34) obtain

$$
\begin{aligned}
\hat{\mathcal{H}}_{2}^{\prime} & =-\frac{e}{m_{e}} \boldsymbol{A} \cdot \hat{\boldsymbol{P}}_{0} \\
& =-\frac{e E_{0}}{2 m_{e} \omega}\left(\mathrm{e}^{i \omega t}+\mathrm{e}^{-i \omega t}\right)\left(-i \hbar \frac{\partial}{\partial x}\right)
\end{aligned}
$$

The fourth part is

$$
\hat{\mathcal{H}}_{3}^{\prime}=\frac{e^{2}}{2 m_{e}} A^{2}=\frac{e^{2} E_{0}^{2}}{8 m_{e} \omega^{2}}\left(\mathrm{e}^{i \omega t}+\mathrm{e}^{-i \omega t}\right)^{2}
$$

This term is purely a change in kinetic energy caused by photon action. It refers to as the third correction term.

So the representation of the Hamiltonian operator is

$$
\begin{aligned}
\hat{\mathcal{H}}= & \frac{1}{2 m_{e}} \hat{\boldsymbol{P}}_{0}^{2}+U(r)+V(r)-\frac{e E_{0}}{2 m_{e} \omega}\left(\mathrm{e}^{i \omega t}+\mathrm{e}^{-i \omega t}\right)\left(-i \hbar \frac{\partial}{\partial x}\right) \\
& +\frac{e^{2} E_{0}^{2}}{8 m_{e} \omega^{2}}\left(\mathrm{e}^{i \omega t}+\mathrm{e}^{-i \omega t}\right)^{2}
\end{aligned}
$$

This representation is relatively complete, including both kinetic energy correction term and potential energy correction term. Usually, to highlight the focus of the problem, textbooks only consider the potential-energy correction term rather than the kinetic energy correction term.

\subsection{Derivation Using Perturbation Theory}

In quantum mechanics, successfully solving the Schrödinger equation for hydrogen atoms obtain a set of eigenvalues and eigenfunctions of the atomic state. Considering a hydrogen atom is in an eigenstate and is affected by an external 
single photon electromagnetic field. According to the usual treatment method, use the $\hat{\mathcal{H}}$ of Equation (40) acting on the assumed wave function, set up the Schrödinger equation, and solve it to obtain the eigenfunction and eigenvalue. But it is too complicated to deal with the problem in this way. It is not easy to solve the new Schrödinger equation. Therefore, quantum mechanics usually regards the electromagnetic field of incident photons as a perturbation in the steady-state. Therefore, using perturbation theory to deal with the collision of a photon with a hydrogen atom is appropriate. To deal with the perturbation problem is to use the approximation method [3] (2) [5] (1). This paper only makes the first-order approximation, ignoring the second-order and higher-order corrections.

Under the steady-state wave function of the hydrogen atom $\Phi_{n}$, there is a relationship

$$
\hat{\mathcal{H}}_{0} \Phi_{n}=i \hbar \frac{\partial \Phi_{n}}{\partial t}=E_{n} \Phi_{n}
$$

The eigenfunction of $\hat{\mathcal{H}}$ is $\Psi$, expanding in eigenfunction $\Phi_{n}$ of $\hat{\mathcal{H}}_{0}$ is

$$
\Psi=\sum_{n} a_{n}(t) \Phi_{n}
$$

Its Schrödinger equation is

$$
i \hbar \frac{\partial \Psi}{\partial t}=\hat{\mathcal{H}} \Psi
$$

Substituting (42) into (43) to get

$$
i \hbar \sum_{n} \Phi_{n} \frac{\mathrm{d} a_{n}(t)}{\mathrm{d} t}+i \hbar \sum_{n} a_{n}(t) \frac{\partial \Phi_{n}}{\partial t}=\sum_{n} a_{n}(t) \hat{\mathcal{H}}_{0} \Phi_{n}+\sum_{n} a_{n}(t) \hat{\mathcal{H}}^{\prime} \Phi_{n}
$$

Applying Equations (41) and (44) can obtain the following equation

$$
i \hbar \sum_{n} \Phi_{n} \frac{\mathrm{d} a_{n}(t)}{\mathrm{d} t}=\sum_{n} a_{n}(t) \hat{\mathcal{H}}^{\prime} \Phi_{n}
$$

Multiply the conjugate wave function $\Phi_{n}^{*}$ of $\Phi_{n}$ on both sides and integrate over the whole space to get

$$
i \hbar \sum_{n} \frac{\mathrm{d} a_{n}(t)}{\mathrm{d} t} \int \Phi_{m}^{*} \Phi_{n} \mathrm{~d} \tau=\sum_{n} a_{n}(t) \int \Phi_{m}^{*} \hat{\mathcal{H}}^{\prime} \Phi_{n} \mathrm{~d} \tau
$$

where

$$
\int \Phi_{m}^{*} \Phi_{n} \mathrm{~d} \tau=\delta_{m n}
$$

The eigenfunction of $\hat{\mathcal{H}}_{0}$ is $\phi_{n}$, thus

$$
\hat{\mathcal{H}}_{0} \phi_{n}=E_{n} \phi_{n}
$$

In this way, expanding the steady-state wave function $\Phi_{n}$ of $\hat{\mathcal{H}}_{0}$ in its eigenfunction $\phi_{n}$ can obtain

$$
\Phi_{n}=\phi_{n} \mathrm{e}^{-\frac{i}{\hbar} E_{n} t}
$$

Substituting it into the integral on the right side of (46) and setting 


$$
\mathcal{H}_{m n}^{\prime}=\int \phi_{m}^{*} \hat{\mathcal{H}}^{\prime} \phi_{n} \mathrm{~d} \tau
$$

Equation (46) becomes

$$
i \hbar \sum_{n} \frac{\mathrm{d} a_{m}(t)}{\mathrm{d} t}=\sum_{n} a_{n}(t) \mathcal{H}_{m n}^{\prime} \mathrm{e}^{i \omega_{m n} t}
$$

where

$$
\omega_{m n}=\frac{1}{\hbar}\left(E_{m}-E_{n}\right)
$$

is the Bohr frequency.

Before the perturbation starts, the system is in the $k$ th eigenstate $\Phi_{k}$ of $\hat{\mathcal{H}}_{0}$, which can obtain the following from Equation (42)

$$
a_{n}(0)=\delta_{n k}
$$

Now consider only the first order approximation, that is, substitute Equation (53) for the $a_{n}(t)$ in the summation sign $\Sigma$ in Equation (42), thus obtained by Equation (51)

$$
i \hbar \frac{\mathrm{d} a_{m}(t)}{\mathrm{d} t}=\sum_{n} \delta_{n k} \mathcal{H}_{m n}^{\prime} \mathrm{e}^{i \omega_{m n} t}=\mathcal{H}_{m k}^{\prime} \mathrm{e}^{i \omega_{m k} t}
$$

Hence, the first-order approximation is

$$
a_{m}(t)=\frac{1}{i \hbar} \int_{0}^{t} \mathcal{H}_{m k}^{\prime} \mathrm{e}^{i \omega_{m k} t^{\prime}} \mathrm{d} t^{\prime}
$$

In this way, the change probability can calculate based on the following:

$$
p=a_{m}^{*}(t) a_{m}(t)=\left|a_{m}(t)\right|^{2}
$$

\subsection{The Operation of the Three Correction Terms of the Hamiltonian Operator}

1) The Operation of the First Correction Term

All quantum mechanics textbooks are very detailed in the first correction term of Hamiltonian operator [3] (3) [5] (2).

Applying the correction value of Hamiltonian operator (33) to Equation (50) can obtain

$$
\begin{aligned}
\mathcal{H}_{1 m k}^{\prime} & =\int \phi_{m}^{*} \hat{\mathcal{H}}_{1} \phi_{k} \mathrm{~d} \tau \\
& =-\frac{1}{2 i} e E_{0} \int \phi_{m}^{*} \hat{x} \phi_{k}\left(\mathrm{e}^{i \omega t}-\mathrm{e}^{-i \omega t}\right) \mathrm{d} \tau \\
& =-\frac{1}{2 i} e E_{0} X_{m k}\left(\mathrm{e}^{i \omega t}-\mathrm{e}^{-i \omega t}\right)
\end{aligned}
$$

where

$$
x_{m k}=\int \phi_{m}^{*} \hat{x} \phi_{k} \mathrm{~d} \tau
$$

Substitute (57-c) into (55) to get

$$
a_{1 m}(t)=\frac{1}{i \hbar} \int_{0}^{t} \mathcal{H}_{1 m k}^{\prime} \mathrm{e}^{i \omega_{m k} t^{\prime}} \mathrm{d} t^{\prime}
$$




$$
\begin{aligned}
& =\frac{e E_{0}}{2 \hbar} \int_{0}^{t} x_{m k}\left(\mathrm{e}^{i \omega t^{\prime}}-\mathrm{e}^{-i \omega t^{\prime}}\right) \mathrm{e}^{i \omega_{m k} t^{\prime}} \mathrm{d} t^{\prime} \\
& =\frac{e E_{0}}{2 \hbar} x_{m k} \int_{0}^{t}\left(\mathrm{e}^{i \omega t^{\prime}}-\mathrm{e}^{-i \omega t^{\prime}}\right) \mathrm{e}^{i \omega_{m k} t^{\prime}} \mathrm{d} t^{\prime} \\
& =\frac{e E_{0}}{2 \hbar} x_{m k} \int_{0}^{t}\left[\mathrm{e}^{i\left(\omega_{m k}+\omega\right) t^{\prime}}-\mathrm{e}^{i\left(\omega_{m k}-\omega\right) t}\right] \mathrm{d} t^{\prime} \\
& =\frac{e E_{0}}{2 \hbar} x_{m k}\left[\frac{\mathrm{e}^{i\left(\omega_{m k}+\omega\right) t}-1}{i\left(\omega_{m k}+\omega\right)}-\frac{\mathrm{e}^{i\left(\omega_{m k}-\omega\right) t}-1}{i\left(\omega_{m k}-\omega\right)}\right]
\end{aligned}
$$

The probability of change is

$$
\begin{aligned}
P_{1}(t) & =a_{1 m k}^{*}(t) a_{1 m k}=\left|a_{1 m}(t)\right|^{2} \\
& =\frac{e^{2} E_{0}^{2}\left|x_{m k}\right|^{2}}{\hbar^{2}} \Gamma_{1}
\end{aligned}
$$

where

$$
\Gamma_{1}=\left[\frac{\sin ^{2} \frac{1}{2}\left(\omega_{m k}-\omega\right) t}{\left(\omega_{m k}-\omega\right)^{2}}+\frac{\sin ^{2} \frac{1}{2}\left(\omega_{m k}+\omega\right) t}{\left(\omega_{m k}+\omega\right)^{2}}-\frac{\cos ^{2} \omega t-\cos \omega_{m k} t \cos \omega t}{\omega_{m k}^{2}-\omega^{2}}\right]
$$

$\left|x_{m k}\right|$ in the above formulas can't get a clear expression. This paper won't go deep into what value to take.

2) The Operation of the Second Correction Term

For the operation of the second correction term of the Hamiltonian operator $\hat{\mathcal{H}}_{2}^{\prime}$, follow the steps from Equation (57) to Equation (61):

Applying Equation (38-b) to Equation (51)

$$
\begin{aligned}
\mathcal{H}_{2 m k}^{\prime} & =\int \phi_{m}^{*} \hat{\mathcal{H}}_{2}^{\prime} \phi_{k} \mathrm{~d} \tau \\
& =-\frac{e E_{0}}{2 m_{e} \omega} \int \phi_{m}^{*}\left(-i \hbar \frac{\partial}{\partial x}\right) \phi_{k}\left(\mathrm{e}^{i \omega t}+\mathrm{e}^{-i \omega t}\right) \mathrm{d} \tau \\
& =-\frac{e E_{0}}{2 m_{e} \omega}\left(\mathrm{e}^{i \omega t}+\mathrm{e}^{-i \omega t}\right) \int \phi_{m}^{*}\left(-i \hbar \frac{\partial}{\partial x}\right) \phi_{k} \mathrm{~d} \tau \\
& =-\frac{e E_{0}}{2 m_{e} \omega}\left(\mathrm{e}^{i \omega t}+\mathrm{e}^{-i \omega t}\right) P_{x m k}
\end{aligned}
$$

where

$$
P_{x m k}=\int \phi_{m}^{*}\left(-i \hbar \frac{\partial}{\partial x}\right) \phi_{k} \mathrm{~d} \tau
$$

Substitute (62-d) into (55) to get

$$
\begin{aligned}
a_{2 m}(t) & =\frac{1}{i \hbar} \int_{0}^{t} \mathcal{H}_{2 m k}^{\prime} \mathrm{e}^{i \omega_{m k} t^{\prime}} \mathrm{d} t^{\prime} \\
& =-\frac{1}{i \hbar} \frac{e E_{0} P_{x m k}}{2 m_{e} \omega} \int_{0}^{t}\left(\mathrm{e}^{i \omega t^{\prime}}+\mathrm{e}^{-i \omega t^{\prime}}\right) \mathrm{e}^{i \omega_{m k} t^{\prime}} \mathrm{d} t^{\prime} \\
& =-\frac{1}{i \hbar} \frac{e E_{0} P_{x m k}}{2 m_{e} \omega} \int_{0}^{t}\left[\mathrm{e}^{i\left(\omega_{m k}+\omega\right) t^{\prime}}+\mathrm{e}^{i\left(\omega_{m k}-\omega\right) t}\right] \mathrm{d} t^{\prime}
\end{aligned}
$$




$$
\begin{aligned}
& =-\frac{1}{i \hbar} \frac{e E_{0} P_{x m k}}{2 m_{e} \omega}\left[\frac{\mathrm{e}^{i\left(\omega_{m k}+\omega\right) t}-1}{i\left(\omega_{m k}+\omega\right)}+\frac{\mathrm{e}^{i\left(\omega_{m k}-\omega\right) t}-1}{i\left(\omega_{m k}-\omega\right)}\right] \\
& =\frac{e E_{0} P_{x m k}}{2 m_{e} \hbar \omega}\left[\frac{\mathrm{e}^{i\left(\omega_{m k}+\omega\right) t}-1}{\left(\omega_{m k}+\omega\right)}+\frac{\mathrm{e}^{i\left(\omega_{m k}-\omega\right) t}-1}{\left(\omega_{m k}-\omega\right)}\right]
\end{aligned}
$$

The probability of change is

$$
\begin{gathered}
P_{2}(t)=a_{2 m k}^{*}(t) a_{2 m k}=\left|a_{2 m}(t)\right|^{2} \\
=\frac{e^{2} E_{0}^{2}\left|P_{x m k}\right|^{2}}{m_{e}^{2} \hbar^{2} \omega^{2}} \Gamma_{2} \\
\Gamma_{2}=\left[\frac{\sin ^{2} \frac{1}{2}\left(\omega_{m k}-\omega\right) t}{\left(\omega_{m k}-\omega\right)^{2}}+\frac{\sin ^{2} \frac{1}{2}\left(\omega_{m k}+\omega\right) t}{\left(\omega_{m k}+\omega\right)^{2}}+\frac{\cos ^{2} \omega t-\cos \omega_{m k} t \cos \omega t}{\omega_{m k}^{2}-\omega^{2}}\right]
\end{gathered}
$$

$\left|P_{x m k}\right|$ in the above formulas can't get a clear expression. This paper won't go deep into what value to take.

The operation results $P_{1}(t)$ and $P_{2}(t)$ of $\hat{\mathcal{H}}_{1}^{\prime}$ and $\hat{\mathcal{H}}_{2}^{\prime}$ are very similar: First, $\left|x_{m k}\right|^{2}$ corresponds to $\left|P_{x m k}\right|^{2}$, further discussion of these two factors will explain the selection rule of atomic energy-level transition. Second, $\Gamma_{1}$ corresponds to $\Gamma_{2}$ in the formulas.

The meaning of these formulas is clear that when $\omega= \pm \omega_{m k}$, the system resonates, so there are two possibilities: In the first case, $\omega=\omega_{m k}$, the atom takes the energy of the photon as $\hbar \omega$ whole absorbed, the photons disappear, and the atomic energy level changes from low energy level $E_{k}$ transition to high energy level $E_{m}$; In the second case, $\omega=-\omega_{m k}$, excited by photons, its energy level changes from high energy level $E_{k}$ transition to low energy level $E_{m}$, emitting a photon with energy $\hbar \omega_{m k}$.

However, if $m=k$, from Equation (52) can obtain $\omega_{m k}=0$, so that Equation (60) becomes

$$
P_{1}(t)=\frac{e^{2} E_{0}^{2}}{\hbar^{2} \omega^{2}}\left|x_{m m}\right|^{2}(1-\cos \omega t)^{2}
$$

Since the photon length is $L=\lambda$, substituting the vibration period $T$ of the photon into the above formula can get

$$
P_{1}(T)=\frac{e^{2} E_{0}^{2}}{\hbar^{2} \omega^{2}}\left|x_{m m}\right|^{2}(1-\cos \omega T)^{2}=0
$$

Similarly, if $m=k$, Equation (65) becomes

$$
P_{2}(t)=\frac{e^{2} E_{0}^{2}}{m_{e}^{2} \hbar^{2} \omega^{2}}\left|P_{x m k}\right|^{2} \sin ^{2} \omega t
$$

Substituting the vibration period $T$ of the photon into the above formula can get

$$
P_{2}(T)=\frac{e^{2} E_{0}^{2}}{m_{e}^{2} \hbar^{2} \omega^{2}}\left|P_{x m k}\right|^{2} \sin ^{2} \omega T=0
$$


Equations (68) and (70)show that that the results of the first correction term $\hat{\mathcal{H}}_{1}^{\prime}$ and the second correction term $\hat{\mathcal{H}}_{2}^{\prime}$ operating on the wave function of the hydrogen atom are that the atom absorbs or emits photons, and its energy level undergoes a transition. At the same energy level, the photon energy cannot be absorbed or emitted.

Therefore, it is more appropriate to combine $P_{1}(t)$ and $P_{2}(t)$ for discussion.

$$
P_{1+2}(t)=P_{1}(t)+P_{2}(t)=\frac{e^{2} E_{0}^{2}}{\hbar^{2}}\left[\left|x_{m k}\right|^{2} \Gamma_{1}+\frac{\left|P_{x m k}\right|^{2}}{m_{e}^{2} \omega^{2}} \Gamma_{2}\right]
$$

Employing Equation (12) can get

$$
P_{1+2}(t)=\frac{\omega^{2}}{c^{2}}\left[\omega^{2}\left|x_{m k}\right|^{2} \Gamma_{1}+\frac{\left|P_{x m k}\right|^{2}}{m_{e}^{2}} \Gamma_{2}\right]
$$

The characteristic of these two effects is that the energy of photon absorbed by the atom either absorbs the whole photon or does not absorb the photon energy at all.

3) The Operation of the Third Correction Term

Now consider the third correction term $\hat{\mathcal{H}}_{3}^{\prime}$ of the Hamiltonian operator. According to Equation (39) and (50), this part of the Hamiltonian operator does not contain parameters about spatial coordinates. Therefore, for function integration over space, the integrand function is constant, so can obtain

$$
\begin{aligned}
\mathcal{H}_{3 m k}^{\prime} & =\int \phi_{m}^{*} \hat{\mathcal{H}}_{3}^{\prime} \phi_{k} \mathrm{~d} \tau \\
& =\frac{e^{2} E_{0}^{2}}{8 m_{e} \omega^{2}}\left(\mathrm{e}^{i \omega t}+\mathrm{e}^{-i \omega t}\right)^{2} \delta_{m k}
\end{aligned}
$$

Substituting it into Equation (55) can get

$$
\begin{aligned}
a_{3 m}(t) & =\frac{1}{i \hbar} \int_{0}^{t} \mathcal{H}_{m k}^{\prime} \mathrm{e}^{i \omega_{m k} t^{\prime}} \mathrm{d} t^{\prime} \\
& =\frac{1}{i \hbar} \frac{e^{2} E_{0}^{2}}{8 m_{e} \omega^{2}} \int_{0}^{t}\left(\mathrm{e}^{i \omega t^{\prime}}+\mathrm{e}^{-i \omega t^{\prime}}\right)^{2} \delta_{m k} \mathrm{e}^{i \omega_{m k} t^{\prime}} \mathrm{d} t^{\prime} \\
& =\frac{1}{i \hbar} \frac{e^{2} E_{0}^{2}}{8 m_{e} \omega^{2}} \int_{0}^{t}\left(\mathrm{e}^{i \omega t^{\prime}}+\mathrm{e}^{-i \omega t^{\prime}}\right)^{2} \mathrm{~d} t^{\prime} \\
& =\frac{1}{i \hbar} \frac{e^{2} E_{0}^{2}}{4 m_{e} \omega^{2}}\left(t+\frac{\sin 2 \omega t}{2 \omega}\right)
\end{aligned}
$$

This formula shows that the absorption of photon energy by an atom is independent of the change of the atom state; that is, it can absorb the fractional energy of photon only under the condition of the same energy level. The probability of absorbing photon energy is

$$
P_{3}(t)=\left|a_{3 m}(t)\right|^{2}=\left[\frac{e^{2} E_{0}^{2}}{4 \hbar m_{e} \omega^{2}}\left(t+\frac{\sin 2 \omega t}{2 \omega}\right)\right]^{2}
$$

The energy absorbed by an atom from a photon is 


$$
\Delta E_{3}(t)=\hbar \omega P_{3}(t)=\frac{\left(e E_{0}\right)^{4}}{16 \hbar m_{e}^{2} \omega^{3}}\left(t+\frac{\sin 2 \omega t}{2 \omega}\right)^{2}
$$

Since the interaction time between photon and atom is equal to the period $T$ of photon vibration [1], using the physical quantity Equation (12) of a single photon and the relationship $\omega T=2 \pi$, from Equation (75), the energy absorbed by the atom from the photon is

$$
\Delta E_{3}=\frac{\pi^{2}}{4} \frac{(\hbar \omega)^{3}}{\left(m_{e} c^{2}\right)^{2}}
$$

Using the classical electron cross-sectional area Equation (15) and photon cross-sectional area Equation (16), the Equation (76-a) can change into

$$
\Delta E_{3}=\frac{3 \pi}{8 \alpha} \frac{\sigma_{T}}{\sigma_{P}} \hbar \omega
$$

where $\alpha$ is the fine structure constant, as Equation (13).

Obviously, from Equation (76) that after a single photon collides with a hydrogen atom, the photon energy absorbed by the hydrogen atom is $\pi / 8 \alpha=53.8$ times of the energy absorbed by the electron when Thomson scattering occurs between the photon and the free electron.

The characteristic of this effect is that the atom absorbs the fractional energy of a photon and will not cause the energy level transition of the atom.

\subsection{Comparison of Results}

Comparing the operation results of the correction terms of the Hamiltonian $\hat{\mathcal{H}}_{1}^{\prime}$, $\hat{\mathcal{H}}_{2}^{\prime}$ and $\hat{\mathcal{H}}_{3}^{\prime}$ is helpful for a deep understanding of their physical meaning.

1) The Difference in Physical Mechanism

Although both $P_{1+2}(t)$ and $P_{3}(t)$ have the meaning of probability, they have different physical meanings.

At present, quantum mechanics is interested in the absorption and emission of photons and the energy level transition of atoms. Therefore, there is often only the Hamiltonian operator correction term $\hat{\mathcal{H}}_{1}^{\prime}$ and $\hat{\mathcal{H}}_{2}^{\prime}$ but not $\hat{\mathcal{H}}_{3}^{\prime}$.

Then, how to understand that the physical meaning of $P_{1+2}(t)$ is the probability of an atom absorbing or emitting a photon, or the probability of an atom's transition.

For example, if $P_{1+2}(t)=0.01$, the atom has a $1 \%$ chance of absorbing the entire photon. That is to say, if 100 photons hit 100 atoms respectively, on average, one of the atoms absorbs one of the photons. The atom either absorbs all the photon energy or does not absorb the photon energy at all. It doesn't mean that each atom absorbs $1 \%$ energy of each photon. If resonance occurs, the probability that the atom absorbs a photon will have a resonance peak.

Therefore, the physical meaning of $P_{1+2}(t)$ is the rate of loss or increase in the number of photons when photons collide with atoms. So, the effect of $\hat{\mathcal{H}}_{1}^{\prime}$ and $\hat{\mathcal{H}}_{2}^{\prime}$ correspond to the attenuation effect of the number of photons. 
Equation (74) is generally ignored in quantum mechanics. An important reason is that compared with the resonance phenomenon represented in Equations (60) and (65), this term is insignificant; Another reason is that it is impossible to derive Equation (76) from Equation (74) under the condition that the size and shape of a single photon are unknown.

Then, what is the effect of the term $\hat{\mathcal{H}}_{3}^{\prime}$ ?

The physical meaning of $P_{3}(t)$ is that an atom fractionally absorbs the energy of a photon, and it does not have an energy-level transition. Since the interaction time between them is equal to the vibration period of the photon, hence $t=T$ in Equation (74), from which can obtain

$$
P_{3}(T)=\frac{\pi^{2}}{4}\left(\frac{\hbar \omega}{m c^{2}}\right)^{2}
$$

For example, if a photon with an energy of $5.1 \mathrm{eV}$ collides with an atom, because $m_{e} c^{2}=0.51 \mathrm{MeV}=5.1 \times 10^{5} \mathrm{eV}$, the energy lost by the photon is only $2.46 \times 10^{-10} \times \hbar \omega$. The rest of the photon energy is not absorbed, and the photon continues to travel forward after reducing fractional of energy.

Generally speaking, the energy loss rate of a photon colliding with an entire atom is insignificant due to $\hbar \omega / m_{e} c^{2} \ll 1$.

Therefore, the physical meaning of $P_{3}(t)$ is the loss rate of photon energy when a photon collides with an atom.

If a distant celestial body emits a large group of independent photons, it will encounter atoms on the way to the Earth. The number of these photons will decrease according to the probability of $P_{1+2}(t)$, and the remaining photons will continue to propagate. The energy of each photon decreases in proportion to $P_{3}(t)$.

It means that the effect of $\hat{\mathcal{H}}_{3}^{\prime}$ corresponds to the energy attenuation effect of a single photon. If the phenomenon that the photon energy decreases on the way of propagation is called redshift, then this paper calls it the quantum redshift effect.

It is a newly discovered redshift effect different from the previously Doppler effect redshift, gravitational redshift, Compton effect redshift, etc.

The correction term of the Hamiltonian operator $\hat{\mathcal{H}}_{3}^{\prime}$ shows that the essence of this effect is that the atom absorbs a fractional of energy of the incident photon and converts it into the kinetic energy of the extranuclear electron. What happens after the electron absorbs this part of the energy? The atomic system does not undergo a transition, but the atom converts this part of energy into low-frequency radio radiation.

The classical electrodynamic method cannot accurately express the two different absorption concepts. Only by applying quantum mechanics can the theory of quantum redshift effect be derived.

2) Meaning in Photometry

A large number of photons in a beam of light, as long as they encounter a large number of atoms on the way of propagation, the number of photons will 
inevitably decrease, resulting in absorption lines on the photometric spectrum. The absorption lines in photometry as the Lya forest is the result of the Hamiltonian $\hat{\mathcal{H}}_{1}^{\prime}$ and $\hat{\mathcal{H}}_{2}^{\prime}$. This effect does not occur during the collision of photons with free electrons. Therefore, the appearance of the absorption line of the photometric spectrum as the Lya forest proves that photons encounter atoms on the way of propagation.

In the photometry of distant luminous objects, absorption lines and redshifts are always accompany each other; that is, any spectrum with redshift must have absorption lines; conversely, any spectra with absorption lines must appear redshift.

Summary:

1) The correction terms of the Hamiltonian operator, $\hat{\mathcal{H}}_{1}^{\prime}, \hat{\mathcal{H}}_{2}^{\prime}$, in the electromagnetic interaction between photons and atoms represents the probability of the number of photons absorbed or emitted by atoms, that is, the rate of increase or decrease in the number of photons, and also represents the probability of the energy level transition of the atom. The effect of decreasing or increasing the number of photons appears as absorption lines and emission lines on the photometric spectrum.

2) The correction term of the Hamiltonian operator, $\hat{\mathcal{H}}_{3}^{\prime}$,in the electromagnetic interaction between photons and atoms represents the absorption rate of single-photon energy or the loss rate of single-photon energy by the atoms. The redshift of the spectral line is evidence of the quantum redshift effect.

\section{Collision between Photon and Helium Atom and Its Analogy}

\subsection{Energy Loss in the Collision of Photon and Helium Atom}

With the successful treatment of the collision between photons and hydrogen atoms, solving the problem of that between photons and other element atoms will be easy. As long as adding a momentum change term caused by the photon vector potential, as in Equation (24), and adding a scalar potential-energy of each extranuclear electron caused by the photon electric field.

When there is no photon electromagnetic field disturbance, the Hamiltonian of the helium atom is

$$
\mathcal{H}_{H_{e 0}}=\frac{\boldsymbol{P}_{10}^{2}}{2 m_{e}}+\frac{\boldsymbol{P}_{20}^{2}}{2 m_{e}}+U_{1}\left(r_{1}\right)+U_{2}\left(r_{2}\right)+U_{12}\left(r_{12}\right)
$$

Due to the emergence of photon electromagnetic field, adding the correction momentum term generated by a vector potential to the momentum of each extranuclear electron, such as Equation (24), and at the same time, adding electric potential energy term generated by a scalar potential to each electron, such as Equation (26), the Hamiltonian of helium atom becomes:

$$
\mathcal{H}_{H e}=\frac{\left(\boldsymbol{P}_{10}-e \boldsymbol{A}\right)^{2}}{2 m_{e}}+\frac{\left(\boldsymbol{P}_{20}-e \boldsymbol{A}\right)^{2}}{2 m_{e}}+U\left(r_{1}\right)+U_{2}\left(r_{2}\right)+U_{12}\left(r_{12}\right)+V_{1}(r)+V_{2}(r)
$$


In the same way as the hydrogen atom problem, the Hamiltonian operator can be divided into four parts:

$$
\begin{aligned}
\hat{\mathcal{H}}_{H e}= & \frac{1}{2 m_{e}}\left(\hat{\boldsymbol{P}}_{10}^{2}+\hat{\boldsymbol{P}}_{20}^{2}\right)+U\left(r_{1}\right)+U_{2}\left(r_{2}\right)+U_{12}\left(r_{12}\right) \\
& +V_{1}(r)+V_{2}(r) \\
& -\frac{e E_{0}}{2 m_{e} \omega}\left(\mathrm{e}^{i \omega t}+\mathrm{e}^{-i \omega t}\right)\left(-i \hbar \frac{\partial}{\partial x_{1}}\right)-\frac{e E_{0}}{2 m_{e} \omega}\left(\mathrm{e}^{i \omega t}+\mathrm{e}^{-i \omega t}\right)\left(-i \hbar \frac{\partial}{\partial x_{2}}\right) \\
& +\frac{e^{2} E_{0}^{2}}{4 m_{e} \omega^{2}}\left(\mathrm{e}^{i \omega t}+\mathrm{e}^{-i \omega t}\right)^{2}
\end{aligned}
$$

The meaning of each part of the Hamiltonian operator represented by this formula is:

The first part (the first row) is the Hamiltonian $\hat{\mathcal{H}}_{\text {He0 }}$ corresponding to Equation (78) when the helium atom is not disturbed by the photon electromagnetic field. The result of the operation on the steady-state wave function of the helium atom is the eigenvalue of the helium atom.

The second part (the second row), $V_{1}(r)+V_{2}(r)$, is the scalar potential energy of two extranuclear electrons of helium atom acted by photon electromagnetic field, which operator is $\hat{\mathcal{H}}_{H e 1}^{\prime}$, and represents the first correction term of the Hamiltonian operator. The result of its operation can explain the number probability of atomic absorption and emission of photons. If the atom is in a state where the photon energy reaches the energy level difference, the probability of absorption or emission increases sharply, resulting in a resonance peak.

The third part (the third row) represents the second correction term $\hat{\mathcal{H}}_{\mathrm{He} 2}^{\prime}$ of the Hamiltonian operator. When it acts on the eigenfunction of the helium atom, two similar to Equation (38-b) appear in the Hamiltonian operator; It can explain the number probability of atomic absorption and emission of photons.

The fourth part (the fourth row) represents the third correction term $\hat{\mathcal{H}}_{\mathrm{He} 3}^{\prime}$ of the Hamiltonian operator of the helium atom. When it operates on the eigenfunction of the helium atom, the value of the operation result is twice that of the Hamiltonian operator of the hydrogen atom.

The method to deal with helium atoms interacting with photons is the same as the method to deal with hydrogen atoms interacting with photons. At first, regarding the eigenvalue and eigenfunction of the Hamiltonian operator $\hat{\mathcal{H}}_{\text {HeO }}$ as known quantities, then treating the other three factors $\hat{\mathcal{H}}_{\mathrm{He} 1}^{\prime}, \quad \hat{\mathcal{H}}_{\mathrm{He} 2}^{\prime}$, and $\hat{\mathcal{H}}_{\mathrm{He} 3}^{\prime}$ as correction terms, rather than using the Hamiltonian operator to directly calculate on the preset wave function to set up the Schrödinger equation and then solve it.

Disregard the first correction term $\hat{\mathcal{H}}_{H e 1}^{\prime}$ to highlight the other point because this term describes the probability of energy level transition due to atomic absorption or emission of photons.

Like the second correction term of the hydrogen atom, the second correction term of the Hamiltonian operator of helium atom, $\hat{\mathcal{H}}_{\mathrm{He} 2}^{\prime}$, will produce two values like Equation (65). 
Like hydrogen atoms, the physical effect of $\hat{\mathcal{H}}_{\mathrm{He} 1}^{\prime}$ and $\hat{\mathcal{H}}_{\mathrm{He} 2}^{\prime}$ operation results is that the photon energy absorbed by the atom either absorbs the whole photon or does not absorb the photon energy at all.

Now, pay special attention to the last term of the Hamiltonian operator, the correction term is as follows

$$
\hat{\mathcal{H}}_{\text {He3 }}^{\prime}=\frac{e^{2} E_{0}^{2}}{4 m_{e} \omega^{2}}\left(\mathrm{e}^{i \omega t}+\mathrm{e}^{-i \omega t}\right)^{2}
$$

Corresponding to the calculation result of the hydrogen atom, Equation (76), the energy lost by a single photon in collision with a helium atom is

$$
\Delta E_{H e}=2 \times \frac{\pi^{2}}{4} \frac{(\hbar \omega)^{3}}{\left(m_{e} c^{2}\right)^{2}}
$$

or

$$
\Delta E_{H e}=2 \times \frac{3 \pi}{8 \alpha} \frac{\sigma_{T}}{\sigma_{P}} \hbar \omega
$$

It shows that the energy lost by a photon in a collision with a helium atom is twice that expressed by the Equations (76-a) or (76-b).

\subsection{Inference}

For an atom of element number $N$, there are $N$ extranuclear electrons. When a photon acts on it, $N$ kinetic energy terms, $N \times\left(\boldsymbol{P}_{0}-e \boldsymbol{A}\right)^{2} / 2 m_{e}$, appear in the Hamiltonian, and $N$ correction terms, as described in Equation (39), appear in the Hamiltonian operator of $N$ electrons. The operation result of each correction one corresponds to the photon energy loss as described in Equation (76).

Reasoning: a photon propagating in the thin intergalactic media, if it is not absorbed by the atom when it collides with the atom with element number $N$, the energy lost by unabsorbed photons is

$$
\Delta E=N \frac{\pi^{2}}{4} \frac{(\hbar \omega)^{3}}{\left(m_{e} c^{2}\right)^{2}}=N \frac{3 \pi}{8 \alpha} \frac{\sigma_{T}}{\sigma_{P}} \hbar \omega
$$

Therefore, the energy lost by a photon is proportional to the number of electrons bound in the atom that it collides with on its propagation path.

Summary:

As the photon collides with the atom, the energy loss of the photon is proportional to the number of extranuclear electrons of the atom encountered. Thus, only the total number of electrons bound in atoms is needed to calculate the energy lost by the photon.

\section{Attenuation Law of Photon Frequency}

\subsection{Propagation of Photons in Thin Atomic Gas}

If there are bound electrons on the path of photon propagation, and their number density is $n_{e}$, the cross-sectional area of a photon is $\sigma_{P}$, then the photon 
travels distance $c \mathrm{~d} t$ in time interval $\mathrm{d} t$, and the swept volume is $\mathrm{d} V=\sigma_{p} c \mathrm{~d} t$, the number of electrons encountered is $\mathrm{d} N_{e}=n_{e} \mathrm{~d} V=n_{e} \sigma_{p} c \mathrm{~d} t$, the photon loses energy every time they collide with electrons, as described in Equation (76), so the energy lost by photons in time interval $\mathrm{d} t$ is

$$
\begin{aligned}
\mathrm{d} \hbar \omega & =-n_{e} \sigma_{P} c \mathrm{~d} t \cdot \frac{3 \pi}{8 \alpha} \frac{\sigma_{T}}{\sigma_{P}} \hbar \omega \\
& =-\frac{3 \pi}{8 \alpha} c \sigma_{T} n_{e} \hbar \omega \mathrm{d} t
\end{aligned}
$$

where $n_{e} \sigma_{P} C$ can be called the collision frequency of a photon and bound electrons.

Equation (84) can express as four equivalent equations

$$
\left\{\begin{array}{l}
\frac{\mathrm{d}(\hbar \omega)}{\mathrm{d} t}+\frac{3 \pi}{8 \alpha} c \sigma_{T} n_{e}(\hbar \omega)=0 \\
\frac{\mathrm{d}}{\mathrm{d} t}\left(\frac{\hbar \omega}{c}\right)+\frac{3 \pi}{8 \alpha} c \sigma_{T} n_{e}\left(\frac{\hbar \omega}{c}\right)=0 \\
\frac{\mathrm{d} \omega}{\mathrm{d} t}+\frac{3 \pi}{8 \alpha} c \sigma_{T} n_{e} \omega=0 \\
\frac{\mathrm{d} v}{\mathrm{~d} t}+\frac{3 \pi}{8 \alpha} c \sigma_{T} n_{e} v=0
\end{array}\right.
$$

When $t=0$, the initial frequency is $v_{0}$, and set

$$
H_{0}=\frac{3 \pi}{8 \alpha} c \sigma_{T} n_{e}
$$

If $n_{e}$ is homogeneous, integrating the Equation (85) can obtain

$$
v=v_{0} \mathrm{e}^{-H_{0} t}=v_{0} \mathrm{e}^{-\frac{H_{0}}{c} r}
$$

where the relationship between distance and time, $r=c t$, is used.

If $n_{e}$ is inhomogeneous, integrating the Equation (85) can obtain

$$
v=v_{0} \mathrm{e}^{-\int_{0}^{r} \frac{H_{0}}{c} \mathrm{~d} r}=v_{0} \mathrm{e}^{-\frac{3 \pi}{8 \alpha} \sigma_{T} \int_{0}^{r} n_{e}(r) \mathrm{d} r}
$$

Representing the above formula as the wavelength changes with time or distance can obtain

$$
\lambda=\lambda_{0} \mathrm{e}^{H_{0} t}
$$

or

$$
\lambda=\lambda_{0} \mathrm{e}^{\int_{0}^{r} \frac{H_{0}}{c} \mathrm{~d} r}=\lambda_{0} \mathrm{e}^{\frac{3 \pi}{8 \alpha} \sigma_{T} \int_{0}^{r} n_{e}(r) \mathrm{d} r}
$$

Equation (87) shows that if a photon encounters atoms when propagating in space, its frequency decreases with distance in exponential attenuation law; In other words, its wavelength increases with distance in exponential growth law.

In the above formula, $H_{0}$ is the attenuation rate of the photon frequency. The speed of light $c$, the fine structure constant $\alpha$ and the electron Thomson scattering cross-sectional area $\sigma_{T}$ are all known physical constants; only the bound electron density $n_{e}$ is an unknown constant. To be sure, $n_{e}$ is inho- 
mogeneous along the path of photon propagation.

\subsection{Damped Motion of A photon}

Photon propagates in intergalactic space with neutral atoms, and its momentum decreases gradually due to the damping effect of the medium.

From Equation (85-2), it is apparent that photons make damping motion in intergalactic space. It shows that the constant $H_{0}$ is the damping coefficient of photon motion. This damping coefficient is also the attenuation rate of photon frequency, energy, and momentum; or the growth rate of wavelength.

Summary:

The collision between photons and atoms on the propagation path causes the photon frequency to decrease with distance in exponential attenuation law or the wavelength increase with distance in exponential growth law. The motion of photons is a damped one. The attenuation rate of photon frequency, i.e., the damping coefficient of the photon, is directly proportional to the number density of extranuclear electrons encountered on the path of photon propagation.

\section{Negation of Hubble's Law}

\subsection{Comparison with Hubble Redshift Formula}

In 1924, Hubble studied a large number of data of extragalactic galaxies and concluded that the redshift is

$$
Z=\frac{\lambda-\lambda_{0}}{\lambda_{0}}=\frac{H_{0}}{c} r
$$

This relationship is called Hubble's law of redshift. Here $H_{0}$ is the Hubble constant, $c$ is the speed of light, and $r$ is the distance from the observed galaxy to the Earth.

Using Equation (87-3), the redshift formula is

$$
Z=\frac{\lambda-\lambda_{0}}{\lambda_{0}}=\mathrm{e}^{\frac{H_{0}}{C} r}-1
$$

Under the first-order approximation, $\mathrm{e}^{\frac{H_{0}}{C} r}=1+\frac{H_{0}}{C} r$, the formula above is given by

$$
Z=\frac{\lambda-\lambda_{0}}{\lambda_{0}}=\frac{H_{0}}{c} r
$$

It is apparent, Hubble's redshift law is only another expression under the first-order approximation of the Equation (87-3).

If interpreting formula (87-3) as classical Doppler effect, then

$$
1+\frac{V}{C}=\mathrm{e}^{\frac{H_{0}}{C} r}
$$

or

$$
V=H_{0} r
$$


This formula is known as Hubble's law.

Due to the redshift effect in the photon-atom collision, the energy of photons decreases with the propagation distance, i.e., the redshift depends on the propagation distance of photons. Therefore, mainstream scholars often attribute this phenomenon to the universe expansion, the so-called cosmological redshift [5] (3). Even they almost express the redshift $Z$ directly as the regression speed of luminaries and intergalactic media. It is a serious misinterpretation. How can the phenomenon caused by the quantum redshift effect of photons take as evidence that the universe is expanding?

In the Big Bang theory, the reciprocal of the photon's damping coefficient, $1 / H_{0}$, is regarded as the universe's age. It is unbelievable.

\subsection{Comparison between Damping Coefficient and Hubble Constant}

$H_{0}$ in Equations (90) (92) is called the Hubble constant in Big Bang cosmology. After nearly a century of measurement, the recognized value of this constant is close to

$$
H_{0}=70 \mathrm{~km} / \mathrm{s} \cdot \mathrm{Mpc}=2.27 \times 10^{-18} / \mathrm{s}
$$

As can be seen from Equation (86), $H_{0}$ is not a constant. No one has ever obtained an exact value in the measurement of nearly a century. Because it is related to the bound electron density on the path of photon propagation, but the atom density in intergalactic space is not uniform, $H_{0}$ is not a constant. However, with the increase of the measurement distance, the measured atom density in intergalactic space tends to average, so $n_{e}$ is also getting closer to the average electron density in cosmology space.

If considering the redshift as caused by the collision between photons and electrons in atoms, the electron density in intergalactic space in the whole universe is given by

$$
n_{e}=\frac{8 \alpha}{3 \pi c \sigma_{T}} H_{0}=0.705 / \mathrm{m}^{3}
$$

The results show that to meet the numerical requirements of the Hubble constant, the average density of bound electrons in intergalactic space is 0.7 electrons per cubic meter. Hydrogen is the most abundant element in nature, accounting for about $71 \%$ by mass; followed by helium, accounting for about $27 \%$; The total atomic mass of the other ones is about $2 \%$.

According to the number of atoms, hydrogen atoms account for $91.2 \%$, helium atoms account for $8.6 \%$, and the total number of one of the other elements is less than $0.2 \%$. Because hydrogen atoms have the minimum mass and are easy to escape from stars and galaxies, the proportion of hydrogen atoms in intergalactic space may be higher than $91.2 \%$. So it can estimate that the mass density of intergalactic matter is about $1.2 \times 10^{-27} \mathrm{~kg} / \mathrm{m}^{3}$.

The actual observations [6] are that the typical density of intergalactic matter 
within the large clusters is $2 \times 10^{-24} \mathrm{~kg} / \mathrm{m}^{3}$ and that outside is $4.6 \times 10^{-31} \mathrm{~kg} / \mathrm{m}^{3}$.

The density of electrons bound to atoms in the intergalactic matter can meet the numerical requirement for the photon energy attenuate rate. But because the mass density in intergalactic space differs by seven orders of magnitude, the attenuate rate of photon energy varies with the path it travels. Therefore, the Hubble constant is not a natural constant and cannot be accurately measured.

Summary:

The attenuation law of photon frequency is evidence of the photon quantum redshift effect. It is unreasonable to regard it as evidence of the expansion of the universe.

\section{Discussion}

\subsection{Classic Problems Worth Discussing}

1 )Will the Image of the Light Source Become Blurred?

Does the redshift caused by photons passing through the intergalactic medium make the light source look fuzzy?

Since the discovery of redshift, some scholars always think that if there is a medium on the way of light propagation, the image of the light source will become blurred.

Of course, there is always a small amount of plasma in the intergalactic medium. So part of photons are scattered by the plasma, but these photons cannot enter the eyes of observers on the Earth. Therefore, the image of the light source will not be blurred.

Because the medium is mainly composed of electrically neutral atoms, however, there is always a part of photons that do not change their propagation direction after the collision.

Because there are positive and negative charges in neutral atoms; for example, in the hydrogen atom, the nucleus has a positive charge $+e$, and the extranuclear electron has a negative one $-e$. In the soft photon electromagnetic field, the proton and the extranuclear electron are acted by an electric field force with equal magnitude and opposite direction simultaneously. So they obtain recoil momentum with the same size and opposite direction simultaneously.

Conversely, the photon is simultaneously acted by the two forces of the nucleus and the extranuclear electron, which are equal in magnitude and opposite in direction. The photon momentum in the direction perpendicular to the propagation direction remains unchanged, and the photon still propagates in the original one.

However, there is plasma near some celestial bodies. When the photons emitted by these objects pass through the plasma, the photons are scattered by the plasma around these objects, so they cannot travel in a straight line. Therefore, their images are very blurry. Some quasars are such confused celestial bodies.

2) Will the Intergalactic Medium Temperature Rise? Will Space Become Bright?

The existence of the intergalactic medium is a fundamental condition for caus- 
ing redshift. Perhaps opponents will refute: will photons collide with atoms in the medium and transfer fractional energy to them, make the medium temperature rise continuously, raise the temperature of cosmic space, and even become bright?

It should explain from two aspects:

First, the intergalactic medium is so thin that it emits light weakly, if at all.

Second, from the principle of atomic absorption of photon energy, the unabsorbed photons only lose a fractional, as described in Equation (76). It will not cause the medium temperature to rise continuously. Because atoms absorb this part of the energy, they radiate it as radio waves, which is why high redshift objects are often radio sources. In the whole space, the light energy emitted by the luminescent body is transformed into radio waves by the medium, so the absorbed energy equals the emitted radio wave energy. Therefore, the temperature of the medium remains unchanged. The photons reduce to that of microwave background radiation, which is invisible light. Finally, the photons disappear gradually.

\subsection{Several Ideas to Explain Redshift Are Worth Discussing}

1) Is the New Tired Light Theory Correct?

The new tired light theory should be the latest theory before the quantum redshift effect.

The scholar Lyndon Ashmore proposed a New Tired Light theory [7] [8]. He believed that the universe is static, and redshifts are produced by an interaction between photons and the electrons in the intergalactic medium.

He derived the Hubble constant as $H_{0}=2 n_{e} h r_{e} / m_{e}$ is very close to Equation (86) derived by this author through quantum mechanics. (Here $n_{e}$ is the electron density in intergalactic space, $h$ is the Planck constant, $r_{e}$ is the classical electron radius, and $m_{e}$ is the electron mass).

He also believes that electron gas condenses to a crystal state when both the Fermi energy and electron temperature are small compared to the energy of mutual repulsion, so the electron gases are arranged into Wigner crystals regularly [9].

Although the main point of the new tired light theory has some correct ingredients, there are at least three difficulties in this view.

The first difficulty was that the author had no idea of the size of the photon. Since any one-dimensional size of a photon in the optical band is much smaller than the Debye length of a plasma, the probability of a photon colliding simultaneously with an electron and proton is extremely low. As a result, as revealed by Compton scattering and Thomson scattering, the propagation direction of photons must change.

The second difficulty is that the intergalactic medium must be highly ionized or composed of an electronic lattice. If so, how to explain the absorption of atoms in intergalactic space? 
The third difficulty is that the electrons of intergalactic matter are composed of electrons arranged in Wigner crystal, which is purely imaginary and too unrealistic.

2) Have There Been Any Other Correct Theories in History?

In the history of physics, there have been Doppler effect theory, tired light theory, photon aging theory, intrinsic redshift theory, gravitational redshift theory, general relativity universe expansion theory, new tired light theory. However, in these views, except that the new tired light theory has some correct ingredients, the other cannot stand theoretical scrutiny.

Electrodynamics has no correct method to explain the origin of redshift. Only quantum mechanics is the right way to explain the origin of redshift. However, if the physical quantities of a single photon structure are unknown in advance, can a concise and clear formula such as Equation (76) be derived only from the theory of quantum mechanics? Quantum mechanics is successful in dealing with the law of atomic energy-level transition and understanding the spectral structure. However, it is unable to deal with the collision between a single photon and an atom.

This paper can accurately deduce the energy loss formula of a single photon in the photon-atom collision, "The size and shape of a single photon" is the theoretical basis. Only by understanding the size, shape, and structure of a single photon can the author deal with the collision between a single photon and a single atom with quantum mechanics and discover the quantum redshift effect.

Summary:

1) The physical mechanism of the redshift caused by the photon-atom collision will neither blur the image of the light source nor cause the universe temperature to rise or even the space to become bright.

2) The theory of the shape and size of a single photon is the basis and premise for solving redshift. The physical mechanism of the redshift has not been correctly explained for a long time in history because of errors in physics theories. The quantum redshift effect is a newly discovered natural law.

\section{Conclusion}

The explanation of the physical mechanism of the origin of the Hubble Redshift by Big Bang cosmologists cannot withstand theoretical scrutiny. Applying the correction of the Hamiltonian operator in quantum mechanics and the derivation of time-dependent perturbation theory conclude that Hubble redshift or cosmological redshift is the result of photon energy loss caused by photon collision with atoms on the path of propagation. On the way of propagation, part of the photons is absorbed by the atoms in a resonance manner, so absorption lines appear on the photometric spectrum. For those unabsorbed photons, the average energy loss caused by each extranuclear electron is $\Delta \mathcal{E}=\pi^{2}(\hbar \omega)^{3} / 4\left(m_{e} c^{2}\right)^{2}=$ $\left(3 \pi \sigma_{T} / 8 \alpha \sigma_{P}\right) \hbar \omega$. In this way, photons frequency decreases with propagation distance in the attenuation law $v=v_{0} \exp \left\{-(3 \pi / 8 \alpha) \sigma_{T} \int_{0}^{r} n_{e}(r) \mathrm{d} r\right\}$, or the wave- 
length of photons increases with propagation distance in the growth law $\lambda=$ $\lambda_{0} \exp \left\{(3 \pi / 8 \alpha) \sigma_{T} \int_{0}^{r} n_{e}(r) \mathrm{d} r\right\}$. This phenomenon is the quantum redshift effect. The so-called Hubble constant is essentially the attenuation rate of photon energy, momentum, and frequency; or the growth rate of wavelength. The Hubble constant can express as $H_{0}=3 \pi c \sigma_{T} n_{e} / 8 \alpha$, which is not a true constant. Its value is directly proportional to the bound electron density in intergalactic space on the path of photon propagation. Hubble constant has nothing to do with the age of the universe. Photon redshift cannot support the theory of the universe's expansion and that of the big bang.

\section{Acknowledgements}

Thanks my family for supporting my scientific research!

\section{Conflicts of Interest}

The author declares no conflicts of interest regarding the publication of this paper.

\section{References}

[1] Xu, Z.L. (2021) Open Access Library Journal, 8, e7179.

[2] Compton, A.H. (1913) The Physical Review, Second Series, 21, 483-502.

[3] Zhou, S.X. (1979) Quantum Mechanics Course. Higher Education Press, Beijing.

[4] Zeng, J.Y. (1984) Modern Physics Series, Quantum Mechanics, Chapter 7. The Motion of Particles in Electromagnetic Field. Science Press of China, Beijing.

[5] Harris, E.G. (1975) Introduction to Modern Theoretical Physics. John Wiley \&Sons, Inc., New York.

[6] He, X.T. (2007) Observational Cosmology, Postgraduate Textbook of New Century Colleges and Universities. Beijing Normal University Press, Beijing.

[7] Ashmore, L. (2016) Journal of High Energy Physics, Gravitation and Cosmology, 2, 512-530. https://doi.org/10.4236/jhepgc.2016.24045

[8] Ashmore, L. (2006) Galilean Electrodynamics, 17.

[9] Ashmore, L. (2019) Journal of High Energy Physics, Gravitation and Cosmology, 5, 181-192. https://doi.org/10.4236/jhepgc.2019.51010 